\title{
Premium Customer Authentication by Using Third Generation Packet Network
}

\author{
Ch.Divya ${ }^{1}$, Dr. P. Govardhan ${ }^{2}$ \\ ${ }^{I}$ (Computer Science and Engineering, Vardhaman College of Engineering, India) \\ ${ }_{2}^{2}$ (Information Technology, Vardhaman College of Engineering, India)
}

\begin{abstract}
In third generation the technology has been developed into new world like as multimedia data transfer rate has been increased from GPRS, $2 G$ to $3 G$ along with these the misuse of data rate is also been increased. To avoid all these kind of scenarios we are going to propose the new ways to reduce the all kinds of vulnerabilities. $3 G$ is the third generation of wireless technologies; It comes with enhancements over previous wireless technologies like high-speed transmission, advanced multimedia access and global roaming. $3 G$ is mostly used with mobile phones and handsets as a means to connect the phone to the internet or other IP networks in order to make voice and video calls, to download and upload data and to surf the net. For real time H.264/AVC video in $3 G$ Wireless environments with benefits shown especially in the presence of link outages. In the 3GPP packet - Switched Streaming Service Rel. 6, H.264/AVC and its RTP Payload format are specified. The RTP payload format allows interleaved transmission of NAL units of H.264/AVC.

Keywords - 3G, HSDPA, Optimal Pricing, Video Streaming, Redistribution.
\end{abstract}

Accepted Date: 25 June 2013

\section{Introduction}

3G follows a pattern of G's that started in the early 1990's by the ITU. The pattern is actually a wireless initiative called the IMT-2000(International Mobile Communications 2000). 3G therefore comes just after $2 \mathrm{G}$ and $2.5 \mathrm{G}$, the second generation technologies. $2 \mathrm{G}$ technologies include, among others, the Global System for Mobile (GSM) - the famous mobile phone technology we use today. 2.5G brings standards that are midway between 2G and 3G, including the General Packet Radio Service (GPRS), Enhanced Data rates for GSM Evolution (EDGE), Universal Mobile Telecommunications System (UMTS) etc.

$3 \mathrm{G}$ is not very cheap, but it is worthwhile for users that need connectivity on the move. Some providers offer it within a somewhat costly package, but most of them have plans where the user pays for the amount of data transferred. This is because the technology is packet- based.

For example, there are service plans where there is a flat rate for the first Gigabyte of data transferred, and a per minute cost for each additional Megabyte. The first thing you require is a device (e.g. a mobile phone) that is $3 \mathrm{G}$ compatible. This is where the name $3 \mathrm{G}$ phone comes from - a phone that has $3 \mathrm{G}$ functionality; nothing to do with the number of cameras or the memory it has. An example is the iPhone 3G. 3G phones commonly have two cameras since the technology allows the user to have video calls, for which a user-facing camera required for capturing him/her.

Unlike with Wi-Fi which you can get for free in hotspots, you need to be subscribed to a service provider to get $3 \mathrm{G}$ network connectivity. We often call this kind of service a data plan or network plan.

Your device is connected to the $3 \mathrm{G}$ network through its SIM card (in the case of a mobile phone) or its $3 \mathrm{G}$ data card (which can be of different types: USB, PCMCIA etc.), which are both generally provided/ sold by the service provider. Through that, you get connected to the internet whenever you are within a $3 \mathrm{G}$ network. Even if you are not in one, you can still use $2 \mathrm{G}$ or $2.5 \mathrm{G}$ services provided by the service provider.

According to a survey on the popularity of mobile devices, almost every person has at least one cell phone in developed countries, and video consumption over mobile devices is an emerging trend. With such a high popularity and the convenient phone-to-phone communication technologies, it is very possible for dataplan subscriber to redistribute the video content without authorization. For example, some users who do not subscribe to the data plan may wish to watch television programs while waiting for public transportation, and some of the them might want to check news from time to time. Hence, these users have incentives to buy the desired video content from neighboring data subscribers if the cost is lower than the subscription fee charged by the service provider. Unlike generic data, multimedia data can be easily retrieved and modified, which facilitates the redistribution of video content. In addition, subscribers also have incentives to redistribute the content with a price higher than their transmission cost, as long as such an action will not be detected by the content owner.

Due to the high mobility, time sensitiveness, and small-transmission-range characteristics of mobile 
devices, each redistribution action only exists for a short period of time and is very difficult to track. Consequently, a better way to prevent copyright infringement is to set a pricing strategy such that no subscriber will have the incentive to redistribute the video.

Wireless technologies are a way for mobile users to make free or cheap calls worldwide and save a lot of money due to the latest telephony applications and services. 3G networks have the advantage of being available on the emitting router. So, a user with a $3 \mathrm{G}$ phone and a $3 \mathrm{G}$ data plan is well- equipped for making free mobile calls. She will only have to download one of the free Applications and install on her mobile phone and start making calls.

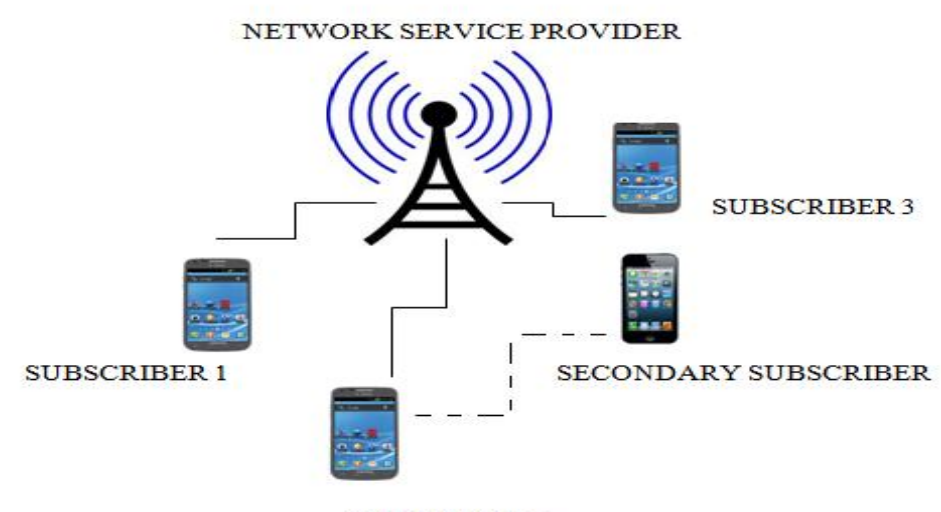

SUBSCRIBER 2

Fig.1 An example of a mobile video stream redistribution network

The rest of this paper is organized as follows:

We introduce the system model in Section II. We analyze the optimal strategies for all users in the redistribution network and prove the existence of the equilibrium when there is only one secondary buyer and We then analyze the mixed-strategy equilibrium for the scenario with multiple secondary buyers, the content owner is also considered as a player who sets the price to maximize his/her payoff but does not prevent the video redistribution among users, In section III we are discussing about future Extension, and Conclusions are drawn in Section IV.

\section{System Model}

In this section, we will introduce the channel, transmission, and video rate- distortion models for the transmission of video streams over wireless networks.

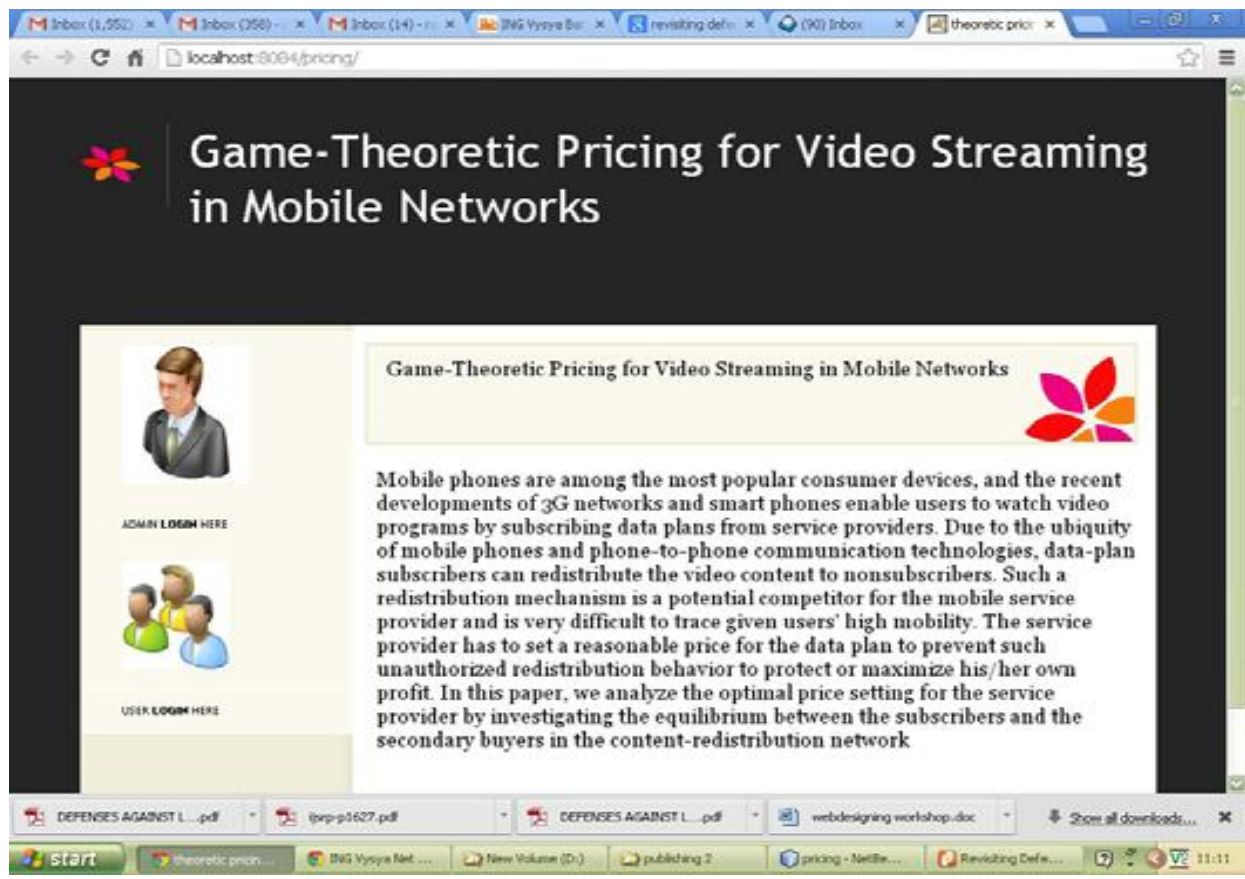

Fig 2. Screen Shot of Video Streaming 


\subsection{G (HSDPA)}

HSDPA stands for High-Speed Downlink Packet Access.

It's a fast $3 \mathrm{G}$ network used by AT\&T and T-Mobile. HSDPA is the fastest of the fast $3 \mathrm{G}$ networks: it is so fast that its often called a $3.5 \mathrm{G}$ network. AT\&T says its HSDPA network can hit speeds of $3.6 \mathrm{Mbps}$ to 14.4Mbps. Real-world speeds typically are slower than that, but HSDPA is still a super-fast network.

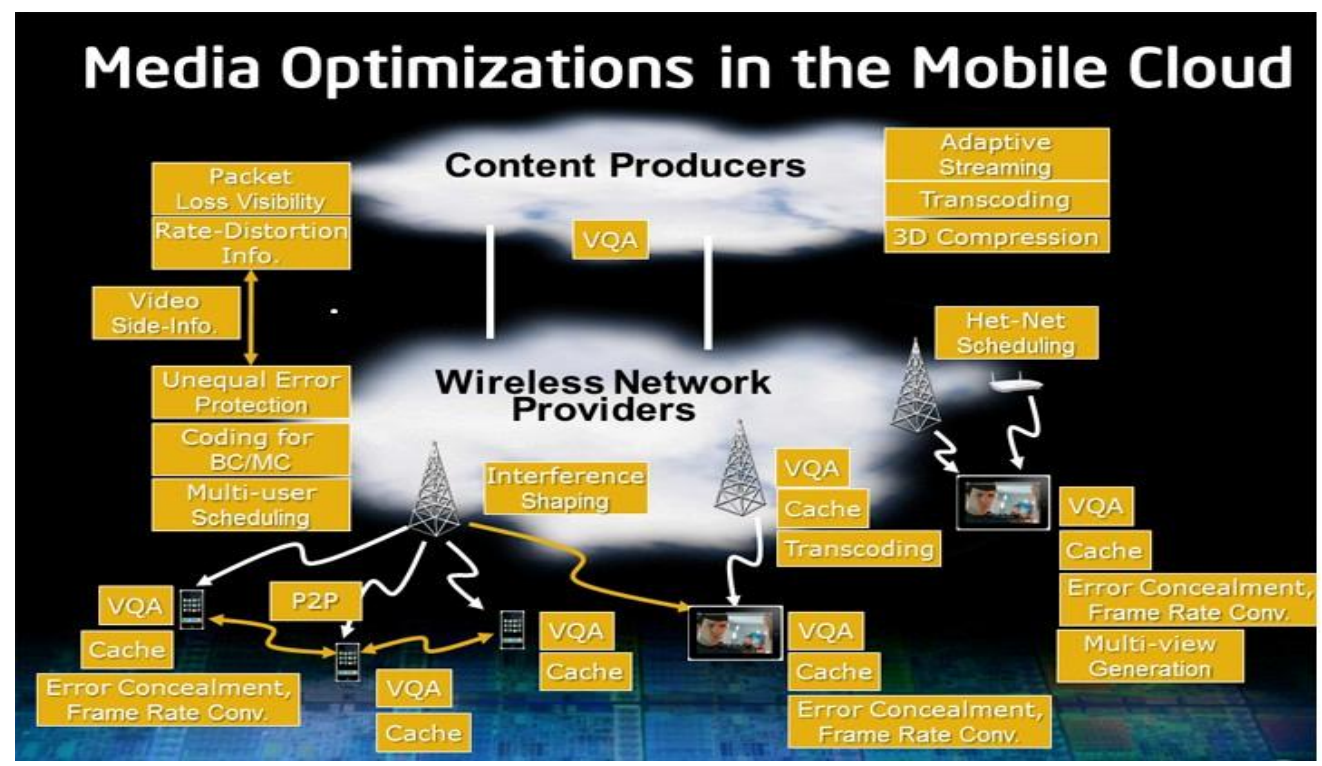

Fig 3. Example of a mobile video streaming redistribution network

\subsection{Third Generation Network}

$3 \mathrm{G}$ services is high-speed access to data and voice services, made possible by the use of a $3 \mathrm{G}$ network. A $3 \mathrm{G}$ network is a high-speed mobile broadband network, offering data speeds of at least 144kilo bits per second (kbps).

For comparison, a dial-up internet connection on a computer typically offers speeds of about 56kbps. If you've ever sat and waited for a web page to download over a dial-up connection, you know how slow that is. 3G networks can offer speeds of 3.1 megabits per second (mbps) or more; that's on par with speeds of offered by cable modems. In day- to-day use, the actual speed of the $3 \mathrm{G}$ network traffic all comes into play. Also known as third- generation service.

\subsection{Premium charges for the content owner}

In the previous sections, we have discussed the equilibrium and the optimal pricing strategy in the video redistribution networks. Our assumption there is that the content owner would like to set price smaller than the equilibrium price in the redistribution network. By doing so, the secondary buyers would have no incentives to purchase the video content from the subscribers and will always subscribe to the data plan from the service provider's goal is not the prevention of video redistribution but rather the maximization of his/her own income. We include the service provider as a player in the game and find his/her optimal strategies.

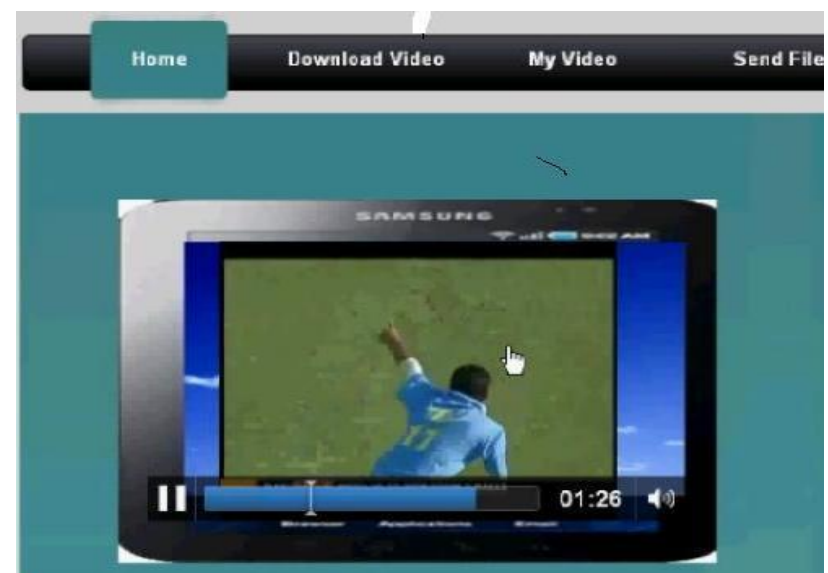

Fig. 4 Optimal video stream price versus qualities of network and streaming service 


\subsection{Premium game model and evolution dynamics}

Here, we model the video pricing problem for the content owner as a no cooperative game, which can be played several times. For example, in practical scenarios, the service provider can always change the price if the total income is below the expectation. Also even when the price is fixed, mobile users can change their mind on whether to subscribe to the data plan or to purchase from other subscribers. Such natural repetition help the players find the equilibrium.

Game stages: in the video pricing game, the first mover's s the service provider, who first sets the price of the video content. Then, mobile users who are interested in the video content decide whether to subscribe to the video streaming service. Since, based on the video content are possible, mobile users also take into consideration the possible payoffs that they can get in the redistribution network when making the decision.

Before the game starts, each user, either a subscriber or the secondary buyer, will declare his/her presence to all other users within his/her presence to all other users within his/her transmission range.

\section{Future Exentension}

The emergence of next generation network (NGN) technologies and increased uptake of bandwidthintensive applications has led to a surgeon in data traffic. Riding on $3 \mathrm{G}$ and $4 \mathrm{G}$ technologies, data services are expected to be the key areas for Indian telecom service providers over the next five years. Mobile data traffic in the country is likely to increase nearly hundredfold by 2015 and consumers will stream nearly 600 hours of video content every second.

\section{Conclusion}

In this paper, we have observed noted the premium charges for mobile video data by analyzing the video redistribution network between data plan subscribers and nonsubscribers. We have first analyzed the equilibrium price of the video stream redistributed by the subscribers given the number of subscribers and secondary buyers.

We have presented a streaming system that utilizes interleaved transmission for real time H.264/AVC video and AAC LC audio in $3 \mathrm{G}$ wireless environments. We have considered audio to be of highest priority. The result provides a guideline for the content owner to prevent the redistribution behavior and to maximize the service provider's payoff. The redistribution behavior has been modeled as a Stackelburg game, and we have analyzed the optimal strategies of both subscribers and secondary buyers. From the simulation results, a secondary buyer will tend to buy more power from subscriber increase, a secondary buyer can obtain a larger utility value, and the payment to each subscriber is reduced due to a more severe competition among the subscriber. Nevertheless, the ser-vice provider should always offer high -quality video stream to prevent the illegal redistribution of video via such itself it has decide the cost. Then only need to forward the content to third parties. This leads to premium Charge for the content owner to maximize his/her total income.

\section{References}

[1] G. Gualdi, A. Prati, and R. Cucciara, "video streaming for mobile video surveillance" IEEE Trants. Multimedia, vol. 10, no. 6, pp.1142-1154, Oct. 2008.

[2] H. Ibaraki, T. Fujimoto, and S. Nakano "Mobile video communications techniques and services" in Proc. SPIE, 1995, vol. 2501, p. 1024.

[3] D. F. S. Santos and A. Perkusich, "Granola: A location and bandwidth aware protocol for mobile video on-demand systems" in Proc. Int Conf. SoftCom. Design, Sep. 2008, pp.309-313.

[4] S. Sudin, A.Tretakov, R. H. R. M. Ali, and M. E. Rusil, "Attacks on mobile networks: An overview of new security challenge" in Proc. Int. Conf. Electron. Design, Dec. 2008, pp. 1-6.

[5] H. Lee, Y. Lee, J. Lee, D. Lee, and H. Shin, "Design of a mobile video streaming system using adaptive spatial resolution control" IEEE. Trans. Consum. Electron. Vol.55, no. 3, pp.1682-1689, Aug. 2009.

[6] T. Schierl, T. Stock hammer, and T. Wiegand, "Mobile video transmission using scalable video coding " IEEE Trans. Circuits Syst. Video Technol., vol. 17, no. 9, pp. 1204-1217, Sep. 2007.

[7] S. Jumisko-Pyykko and J. Hakkinen, "Evaluation of subjective video quality of mobile devices" in Proc. $13^{\text {th }}$ Annu. ACM Int. Conf. Multi-media, 2005, p.538. 